\section{AB003. Stereotactic body radiation therapy vs. pulmonary resection}

\author{
Michael Lanuti \\ Division of Thoracic Surgery, Massachusetts General Hospital, Boston, \\ MA, USA
}

Correspondence to: Michael Lanuti, MD. Division of Thoracic Surgery, Massachusetts General Hospital, 55 Fruit Street, Founders 7, Boston, MA 02114, USA. Email: mlanuti@mgh.harvard.edu.

\begin{abstract}
Pulmonary resection remains the accepted standard of care for early stage non-small cell lung cancer (NSCLC) in patients with adequate cardiopulmonary reserve. Radiation therapy, and in particular stereotactic body radiation therapy (SBRT), has traditionally been recommended as curative therapy for medically unfit patients who harbor early stage lung cancer. As a consequence of the rising success of SBRT in patients with medically inoperable early stage NSCLC (evidenced by Radiation Therapy Oncology Group trials 0236 and 0915), there has been keen interest in exploring this modality in operable patients. SBRT to lung is often very well tolerated, thus making it very attractive to patients. The risk of series complications in carefully selected patients is low. Equipoise among surgeons and the medical community for the use of SBRT as curative therapy for patients eligible for surgery has been limited, thus contributing to the poor accrual and early closure of 3 randomized trials attempting to directly compare SBRT to surgery in operable patients. These trials included the STARS trial (Study of Anastrozole and Radiotherapy Sequencing), the ROSEL trial [Either Surgery or Stereotactic Radiotherapy for Early-Stage (IA) Lung Cancer] and the American College of Surgeons Oncology Group (ACOSOG) Z4099 trial. No preliminary data is available from the ACOSOG trial. The STARS and ROSEL trial randomized operable patients with tumors $<4 \mathrm{~cm}$ (cT1-T2a) and required invasive mediastinal staging in patients harboring radiographically suspicious lymph nodes. Sublobar resection was not permitted in these trials. Since patient accrual was limited ( $\mathrm{n}=58$ total patients) the two trials were combined in a controversial publication that showed improved overall survival and decreased toxicity with SBRT compared to surgery. This has spawned additional randomized trials including the STABLE-MATES trial comparing sublobar resection and
\end{abstract}

SBRT in patients with stage I NSCLC (NCT02468024) with an estimated enrollment of 258 patients, a Veterans Affairs Lung Cancer or Stereotactic Radiotherapy (VALOR) trial (NCT02984761) with estimated enrollment of 670 patients, and lastly, a phase II United Kingdom sponsored SABRTooth study (NCT02629458) that is enrolling up to 54 patients. The lack of randomized data has stimulated multiple publications that retrospectively examine these modalities in single center case series and more recently in larger data sets derived from the National Cancer Database (NCDB), the Surveillance, Epidemiology, and End Results (SEER)-Medicare linked database and the United States Veterans Affairs Health Care System. These retrospective analyses are clearly limited by patient selection bias and different levels of staging accuracy. Propensity matched retrospective series showed that overall survival was no different at 1 year but favored surgery at 3 years. Cancer-specific survival (clearly a better outcome measure particularly in elderly patients receiving SBRT) was no different compared to surgery, nor was local failure, or the risk of developing distant metastases. Collectively, the studies that used large data with statistical matching demonstrated a survival advantage of surgery (namely lobectomy) over SBRT for stage I NSCLC but raised awareness of the increased post procedure morbidity and mortality for surgery compared to SBRT. Interestingly, sublobar resection versus SBRT in these large data sets had mixed results with some showing survival improvement with surgery and some showing no survival difference. Surgery appears superior at later time points. In a phase II ablate and resect human clinical trial (MISSILENSCLC, NCT02136355) for early stage (T1-T2) NSCLC, 35 patients underwent neoadjuvant SBRT using 54-60 Gy in 3-8 fractions followed by surgery at an interval of 10 weeks. The authors reported a $60 \%$ pathologic complete response rate, invoking many questions about local control of SBRT. Assessment of local control after SBRT remains the single most challenging real-world clinical problem. The lung injury post ablation is heterogenous and requires very careful observation with imaging modalities that lack sensitivity and specificity for recurrence. At present, the American Society of Radiation Oncology does not recommend SBRT for operable patients with early stage NSCLC outside of a clinical trial.

Keywords: Stereotactic body radiation therapy (SBRT); lung cancer; lobectomy; survival

doi: 10.21037/shc.2019.AB003

Cite this abstract as: Lanuti $M$. Stereotactic body radiation therapy vs. pulmonary resection. Shanghai Chest 2019;3:AB003. 